\title{
High tibial osteotomy
}

\author{
Davide Edoardo Bonasia • Giorgio Governale • \\ Simone Spolaore $\cdot$ Roberto Rossi $\cdot$ Annunziato Amendola
}

Published online: 17 August 2014

(C) Springer Science+Business Media New York 2014

\begin{abstract}
High tibial osteotomy (HTO) is a widely accepted and performed procedure to treat medial knee arthrosis. The aim of this review is to evaluate the different surgical options in medial knee arthrosis, focusing on indications, patient's selection, long-term follow-up and survival analysis of HTO. Comparison and pooling of results are challenging because of different evaluation systems, small cohort number, and different surgical techniques. No differences have been described between opening and closing wedged HTO in terms of outcomes. Excellent early survivorship and good clinical outcomes were reported also with concomitant procedures. Correct indications, preoperative workup/planning, and technique selection are essential in achieving good results. The choice between opening and closing wedge osteotomy, graft selection in opening wedge HTO, comparison between HTO and unicompartmental knee arthroplasty, and the results of revised HTO to total knee replacement are currently under debate and will be discussed in the present review.
\end{abstract}

Keywords High tibial osteotomy $\cdot \mathrm{HTO} \cdot$ Medial arthritis · Knee $\cdot$ Opening wedge $\cdot$ Closing wedge

D. E. Bonasia $(\bowtie) \cdot G$. Governale $\cdot$ S. Spolaore

Department of Orthopaedics, University of Torino, CTO Hospital,

Via Lamarmora 26, 10128 Torino, Italy

e-mail: davidebonasia@virgilio.it

R. Rossi

Department of Orthopaedics, University of Torino, Mauriziano

"Umberto I" Hospital, Torino, Italy

A. Amendola

Department of Orthopaedics and Rehabilitation, University of Iowa

Hospitals and Clinics, Iowa City, IA, USA

\section{Introduction}

High tibial osteotomy (HTO) is a widely accepted procedure to treat varus alignment of the knee associated with medial compartment arthrosis/overload.

Valgus producing proximal HTO shifts the mechanical axis of the lower limb from the medial compartment laterally, decreasing medial contact pressure, and contact area. Medial compartment unloading leads to reduced pain, improved function, and the potential reduction of cartilage degeneration.

Correct patients' selection is fundamental in achieving good results.

Many techniques have been described (ie, closing wedge, opening wedge, dome, and "en chevron" osteotomies), but opening (medial) and closing (lateral) wedge HTO are the most commonly used.

Opening wedge HTO has recently gained popularity since it does not require a fibular osteotomy, common peroneal nerve dissection, disruption of proximal tibiofibular joint, and bone stock loss as opposed to closing wedge HTO. In addition, opening wedge HTO allows for multiplanar correction and easier subsequent total knee replacement (TKR).

Disadvantages associated with medial opening wedge HTO include the need of bone graft and the risk of nonunion, collapse, or loss of correction $[1 \bullet, 2,3 \bullet]$.

The aim of this paper is to review the indications, clinical results, fixation techniques, and new tendencies in HTO.

\section{Indications HTO}

Selection of the ideal patient is the key factor in achieving good results with HTO.

Conditions related with poor outcomes are: severe joint destruction ( $\geq$ Ahlback grade III), $\geq 65$ years of age, $<90^{\circ}$ of $\mathrm{ROM}, \geq 15^{\circ}$ of flexion contracture, joint instability together 
with $\geq 1 \mathrm{~cm}$ lateral tibial thrust, $\geq 20^{\circ}$ of correction, rheumatoid arthritis, and advanced patellofemoral arthritis [4-7].

The influence of Body Mass Index (BMI) remains a controversial factor [7].

Young patients ( $<60$ years of age), with isolated medial osteoarthritis, with good range of motion, and without ligamentous instability are the ideal candidates for an HTO.

Although originally instability was considered as a contraindication for HTO, recently HTO, with or without combined ligamentous reconstruction, has raised in popularity for the treatment of: chronic knee instability, ligament reconstruction failure, and medial arthrosis associated with knee instability.

\section{Overall results}

We report here a short review of the literature presenting the state of the art regarding opening and closing wedge HTO (Fig. 1).

Survivorship analysis is the preferred method to assess quality and durability of the results because it does not exclude inadequate follow-up, loss to follow-up, and patient's death.

\section{Opening wedge HTO}

Shallberger et al retrospectively reviewed the long-term outcomes of opening and closing wedge osteotomies in 54 patients. The median follow-up was of 16.5 years, 13 patients (24\%) underwent TKR. Osteotomy survival was $98 \%$ after 5 years, $92 \%$ after 10 years, and $71 \%$ after 15 years. It was also shown that: (1) osteotomy survivors, despite the mean age of 61 years, had a high level of activity; (2) no significant differences in survival and outcome scores between opening and closing wedge HTO were found. The authors noticed low levels of pain as indicated in the Visual Analogue Scale, a high Satisfaction Index and good values in the KOOS and WOMAC scores. The authors, in the radiological follow-up, showed a slight osteoarthritis progression (according to the Kelleren and Lawrence classification) despite good correction was achieved. They concluded that HTO is a successful treatment in unicompartmental knee degeneration associated with varus alignment in active patients [8].

Niemeyer et al evaluated the 3-year clinical results of 69 patients with medial compartment arthrosis treated with open wedge HTO, using internal plate fixation (TomoFix, Synthes) in a prospective, observational case series. Additional surgical procedures were performed in 55 patients (ie, microfractures, autologous chondrocyte implantation, partial medial meniscectomy, ACL reconstruction). The authors evaluated knee function with significant improvement of International Knee Documentation Committee (IKDC) and Lysholm scores. They noted function improvement between 12 and

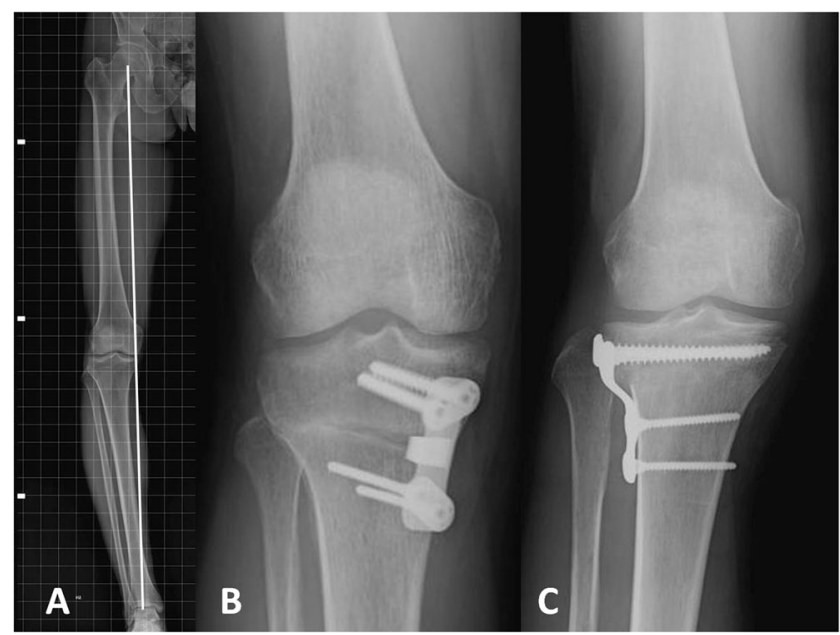

Fig. 1 A Preoperative AP view long leg x-ray showing varus malalignment (white line $=$ mechanical axis). B AP view of the right knee after opening wedge HTO. C AP view of the right knee after closing wedge HTO

24 months and between 24 and 36 months, the latter probably due to implant removal. Damage severity of the medial cartilage was not decisive on clinical outcomes and partial thickness defects of the lateral compartment were well-tolerated as well. Discomfort related with the implant was the most common complication $(40.6 \%)$ [9•].

Bode et al evaluated 62 patients after HTOs with internal plate fixator (TomoFix, Synthes), using IKDC score, Lysholm score, and Survival analysis. A $96 \%$ survival rate after a mean follow-up of over 5 years was observed. IKDC and Lysholm improved significantly when compared with preoperative values. The overall complication rate was $8.6 \%$ [10].

Shröter et al performed a prospective clinical and radiographic evaluation after 35 open wedge HTOs using the Position HTO Plate (Aesculap, Tuttlingen, Germany), without bone augmentation. They concluded that this specific type of plate cannot be recommended, due to an overall complication rate of $34 \%$, plate-related complication rate of $23 \%$, and significant loss of correction between 2 and 6 months of follow-up. However, clinical outcomes improved significantly after 12 months (HSS, Lysholm-Gillquist, IKDC, Tegner activity level scores). Preoperative scores were excellent in $23 \%$ of the cases, good in $47 \%$, fair in $20 \%$, and poor in $10 \%$. Postoperatively, $63 \%$ were excellent, $31 \%$ good, and $6 \%$ fair [11].

Hernigou et al followed up for an average of 10 years on 53 knees treated with proximal opening-wedge HTOs for large varus deformity and osteoarthritis of the medial compartment. A buttress plate and a porous betatricalcium phosphate wedge were used. After 10 years, $81 \%$ had excellent or good results. The best results were obtained in knees with a postoperative hip-knee-ankle angle ranging from $183^{\circ}$ to $186^{\circ}$. The authors concluded that this is the correction to be achieved during the HTO [12]. 
DeMeo et al in a prospective study evaluated 20 consecutive patients affected by varus gonarthrosis and treated with medial opening-wedge HTOs, using the Puddu plate and allograft augmentation. Patients were evaluated preoperatively, at 2 and 8 years of follow-up with radiographs, subjective scores, and knee scores (Lysholm and Hospital for Special Surgery [HSS] scores). The overall survivorship at 8 years was $70 \%$, and $57 \%$ of the patients reported subjective good to excellent results. At final follow-up, 5 patients $(25 \%)$ were converted to TKR. Both Lysholm and HSS knee scores improved at 8 years of follow-up [13].

LaPrade et al conducted a case series study to evaluate the outcome of opening wedge HTOs performed in 47 young and middle-aged patients $(<55$ years) to treat symptomatic medial compartment osteoarthritis of the knee. The survival rate of the osteotomy was $94 \%$ at a mean follow-up of 3.6 years with only 3 failures. They found a small but statistically significant increase in postoperative tibial slope and showed that postoperative mechanical axis passing close to the lateral tibial eminence resulted in a significant improvement of the scores. Patellar height was decreased in $74 \%$ of the patients [14].

Bonasia et al retrospectively evaluated the prognostic factors of 99 medial opening wedge HTOs in 84 patients (mean age 55 years) affected by symptomatic medial knee overload/ arthritis, with a mean follow-up of 51.5 months. They used the Knee Society score (KSS) and the Western Ontario and McMaster Universities Osteoarthritis Index score (WOMAC). Clinical and functional scores significantly improved after surgery. The variables significantly related with a poor outcome were age $>56$ years and postoperative knee flexion $<120^{\circ}$. The variables significantly related with a good outcome were Ahlbäck grade 0 arthritis of the medial compartment and excellent preoperative Knee Society score. The Kaplan-Meier analysis showed a survival rate of $98.7 \%$ at 5 years and $75.9 \%$ at 7.5 years [15•].

Niinimäki et al evaluated survivorship of HTO at a national level using the Finnish registry. They studied 3195 HTOs performed between 1987 and 2008. Kaplan-Meier analysis revealed an overall survivorship of $89 \%$ at 5 years and $73 \%$ at 10 years, with revision to TKR as an endpoint. Females and patients $>50$ years old had worse survivorship than males or patients $\leq 50$ years old $\left[16^{\bullet}\right]$.

\section{Closing wedge HTO}

Flecher et al reviewed 301 closing wedge HTOs at a minimum 12-year follow-up synthesized with Blount staple and an AO half-tube plate with 3 screws. The mean patient age was 42 years. They analyzed patient satisfaction, radiographs, and survivorship. Survival rate was $92 \%$ at 10 years and $85 \%$ at 20 years with revision as an endpoint. Knee function was considered satisfactory by $77 \%$ of patients after an average follow-up of 18 years and younger patients had better long-term results. Twenty-three of 31 knees ( $74 \%$ ), classified as Ahlback Grade 3 preoperatively, required revision at a mean follow-up of 16 years. They concluded that ideal candidates for closing wedge HTOs are patients with minor medial tibiofemoral osteoarthritis (Ahlback grade $<3$ ) and younger than 50 years with active lifestyle [17].

Akizuki et al conducted a prospective study on 94 patients (118 knees) treated with closing wedge HTOs and internal fixation. Failure was defined as revision to TKR and HSS score $<70$ points. Survivorship was $97.6 \%$ at 10 years and 90.4 at 15 years from surgery [18].

Gstöttner et al studied long-term follow-up after closing wedge HTO fixed with staples. They investigated 134 HTOs in 111 patients and they found a survivorship of $94 \%$ after 5 years, $79.9 \%$ after 10 years, $65.5 \%$ after 15 years and $54.1 \%$ after 18 years. The authors showed that age influenced the survival rate and the hazard of failure increased by $5 \%$ per year of age. Complications rate was $28.4 \%$ [19].

Hui et al determined survival rate of closing wedge HTO in a large case series. They performed lateral closing wedge HTOs for medial compartment osteoarthritis between 1990 and 2001 in 455 patients. Between 2008 and 2009, patients were contacted by phone, and assessment included incidence of further surgeries, current body mass index (BMI), Oxford Knee Score, and British Orthopaedic Association Patient Satisfaction Scale. The authors described a survival rate of $95 \%$, $79 \%$, and $56 \%$ at 5,10 , and 15 years of follow-up, respectively. They also described better results in patients under 50 years of age, with BMI less than 25, and ACL deficiency [20].

The aim of the study conducted by Efe et al was to elucidate the outcome and assess the influence of risk factors on long-term results of closing wedge HTO.

They retrospectively studied 199 patients with a mean follow-up of 9.6 years. Failure was defined as the need for revision to TKR. They recorded 39 complications. Thirty-six (16\%) HTOs were converted to TKR. The 5-year, 9.6-year, and 15-year survival rates, as determined by Kaplan-Meier analysis, was $93 \%, 84 \%$, and $68 \%$, respectively. Fifty-four patients had excellent results, 74 good, 51 fair, and 20 poor. Pain was absent in 74 patients, mild in 66, and severe in motion but not at rest in 58. A significant preoperative risk factor for HTO failure was osteoarthritis Kellgren-Lawrence grade $>2$ [21].

The overall results are summarized in Table 1.

\section{Opening vs closing wedge HTO}

As shown, good and excellent results can be achieved with both techniques at short- and mid-term follow-up but the results deteriorate over time. 


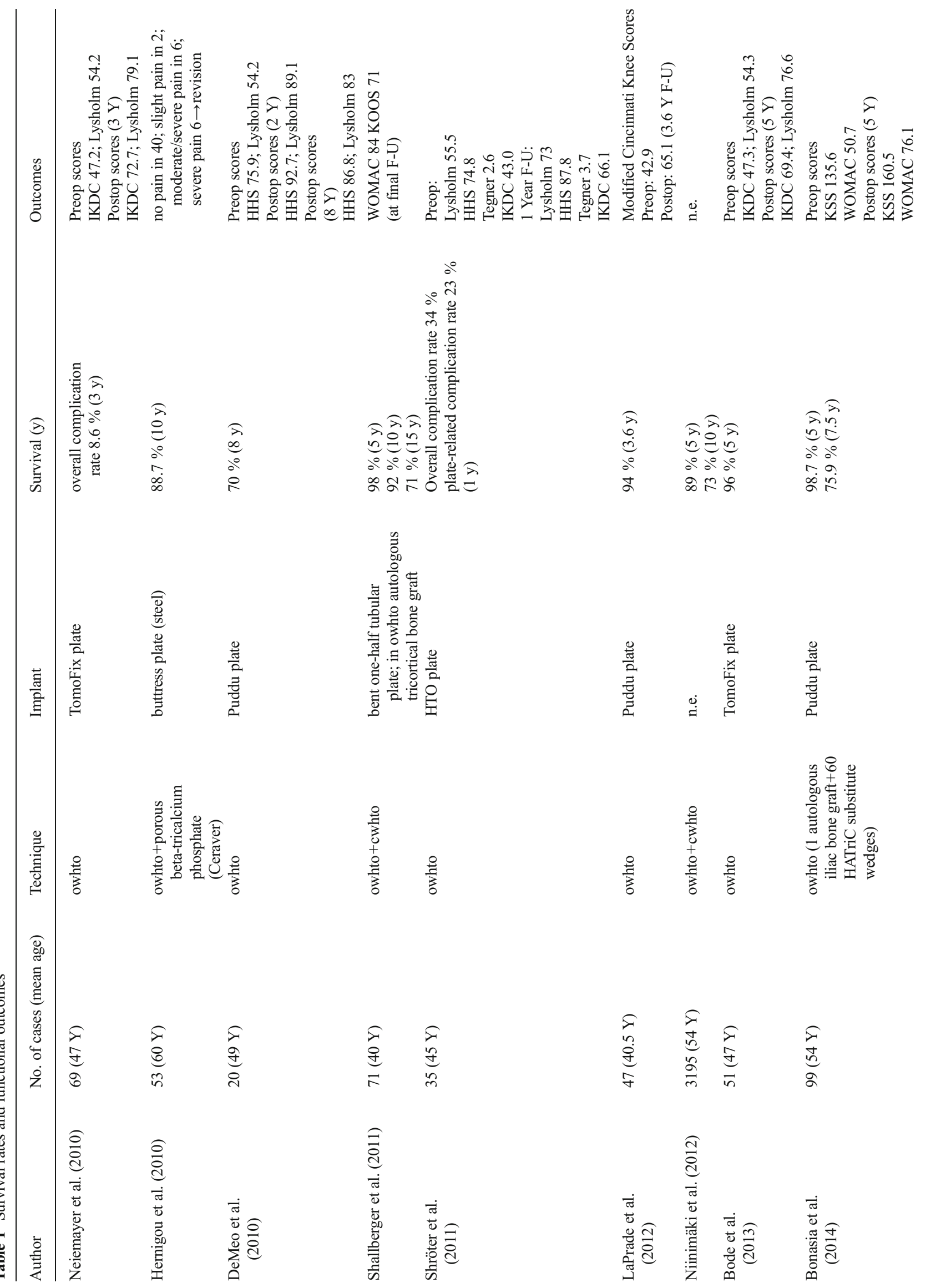




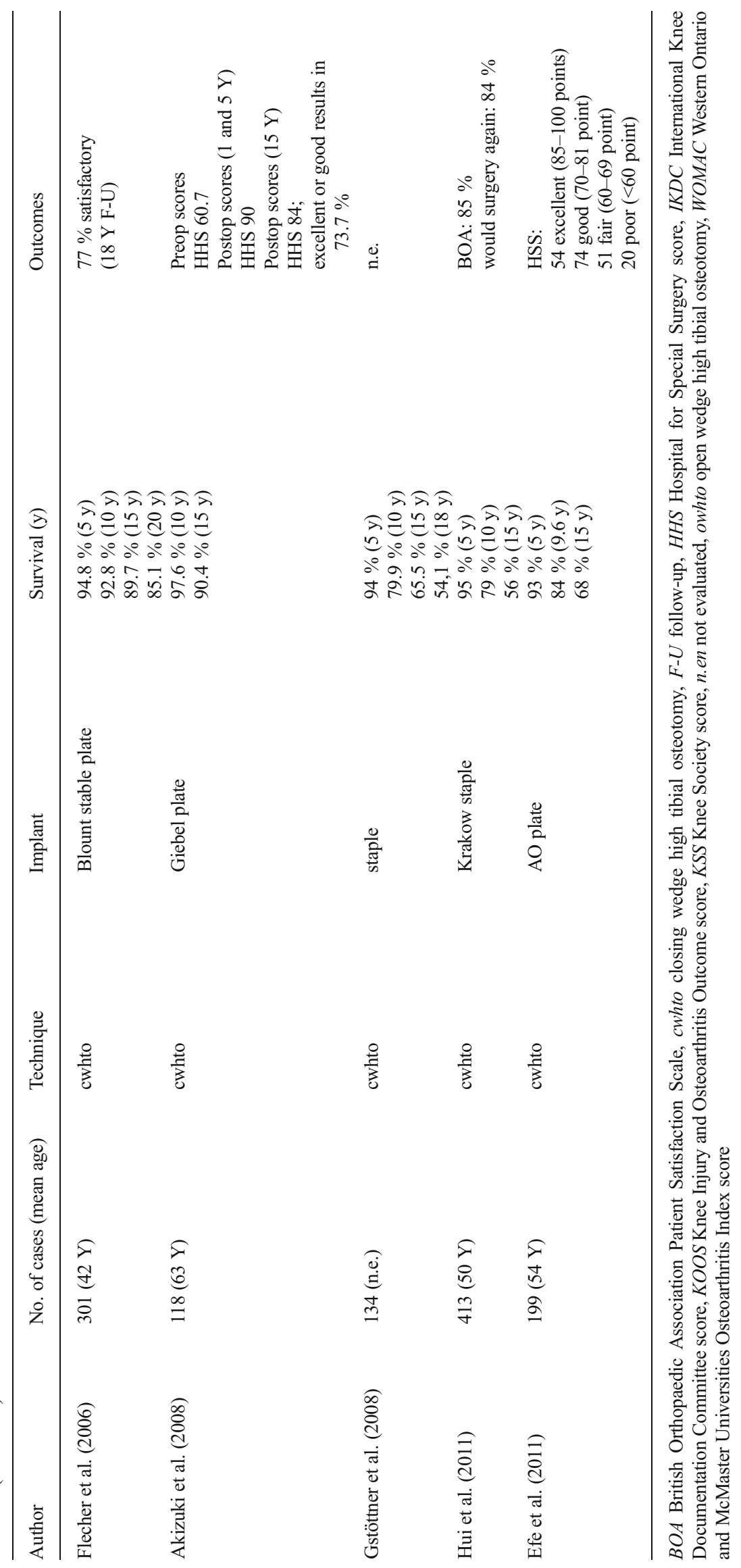


The closing wedge HTO was first described by Jackson and Waugh in 1961 [22] and then further popularized by Coventry in 1965 [23].

Lateral closing wedge HTO has been considered for a long time the gold standard in treating isolated medial knee osteoarthritis in young and active patients.

The advantages of this technique can be summarized in quicker healing, shorter time to weight-bearing, and reduced risk of patella baja compared with opening wedge HTO. In addition, there is no need for bone graft or synthetic bone substitute.

However, closing wedge HTO implies fibular osteotomy or proximal tibiofibular joint disruption, lateral muscle detachment, peroneal nerve dissection, bone stock loss, and more demanding subsequent TKR.

A recent review conducted by Atrey et al reported a pooled complication rate between $5 \%$ and $34 \%$ with an average of $15.2 \%$ (including infections, thromboembolic events, fractures, nerve and vascular injury, nonunion, or delayed union) [24].

In order to prevent injury to the common peroneal nerve, improve correction and union, Huang et al recently described a modified closing wedge HTO technique in 75 knees (46 patients). The osteotomy was performed at the distal third of the tibial tuberosity and an L shaped LCP plate was used. They concluded that this modified technique is efficient and safe for the correction of tibia vara in young patients [25].

For the above mentioned disadvantages of closing wedge HTO and for the possibility of multiplanar correction, opening wedge HTO became a widely accepted alternative option.

However, this technique is also not free from complications, and these include: possibility of collapse, nonunion, loss of correction with or without hardware failure [11,26], and the necessity of bone graft with possible donor site morbidity (in case of autograft) or disease transmission (in case of allograft).

A recent meta-analysis by Smith et al included 12 papers reporting 9 clinical trials comparing 324 opening wedge HTOs vs 318 closing wedge HTOs. They found no significant differences between the 2 groups in terms of clinical outcomes or complications. The authors reported increased tibial slope and higher risk of patella baja in the closing wedge group. On the other hand, closing wedge HTOs resulted in decreased tibial slope [27].

Harris et al in a systematic review determined survivorship and clinical outcomes of HTO isolated and combined with other procedures (ie, articular cartilage surgery, medial meniscus allograft transplantation). The authors also compared survivorship and clinical outcomes of opening and closing wedge HTOs. They concluded that at 2 years of follow-up, survivorship was significantly better in the opening wedge group $(98.7 \%)$ compared with the closing wedge group $(96.7 \%)$. At all other time points, with or without combined procedures, there was no significant survival difference between the 2 techniques. Radiographically, they also confirmed that tibial slope was increased and patellar height decreased with opening wedge HTO. Conversely, tibial slope was decreased and patellar height increased with closing wedge techniques [2].

Similar results were obtained in a randomized controlled trial by Brower et al, which showed improvement in knee function and pain without significant differences between closing and opening HTOs [28].

All the authors agree that pooling the results is challenging and further studies are necessary to determine the superiority of one procedure over the other.

\section{Augmentation and type of fixation in opening wedge HTO}

Nonunion is one of the main risks in open wedge HTO, with an incidence ranging from $0.7 \%$ to $4.4 \%$ [26].

Many techniques have been developed to fill the void created with an opening wedge HTO and to achieve reliable fixation, with early weight bearing.

Iliac crest autograft or tricortical allograft remaining the most commonly used grafting modalities [29].

Osteoconductive, osteoinductive, and osteogenic properties are the main advantages of autografts. However, the rate of minor and major complications at the iliac crest donor site has been shown to range from $9 \%$ to $39 \%$ and from $0.76 \%$ to $25 \%$, respectively [29].

To avoid these complications a new technique using a reamer-irrigator-aspirator has been described by Seagrave et al in a small case series, with good results at 12 months of follow-up [30].

Allograft has lower osteoinductive properties and entails virtual disease transmission risks.

Kuremsky et al compared allograft vs autograft using a medial opening wedge HTO plate system. They had a 6-fold higher failure rate using allograft and they also noted that lateral cortical disruption associated with allograft augmentation led to failure in $53 \%$ of the cases. The authors recommended autograft or allograft with 6 weeks of nonweight bearing in case of lateral disruption or large corrections [31].

Synthetic bone substitutes (hydroxyapatite, $\beta$-tricalcium phosphate, a combination of both, bone cement) showed lower resistance than macroporous ceramics to compressive load [32].

Bone cement as a spacer has been described [33] but in order to achieve a more biological regeneration is not recommendable [32].

The use of PRP, bone stromal cells, and growth factors showed encouraging results and improvement in osteotomy union in association with autograft, allograft, and bone substitutes $[32,34,35]$. 
However, there is no evidence whether PRP and synthetic augmentation lead to superior union rates compared with autologous iliac crest graft [32, 36, 37].

Stable fixation is fundamental in achieving good results by maintaining the desired correction and allowing early weight bearing.

Numerous studies described the biomechanical properties of different implants [38-41].

Although long-locking plates showed stronger properties, permit early weight bearing, and seem to be more reliable [42] compared with other fixation devices, they are bulky and frequently require removal [32]. Smaller spacer plates have lower mechanical properties compared with long-locking plates, but can still be reliable and are less likely to be removed.

New PEEK (polyetheretherketone) devices have recently been introduced on the market [43], but their reliability and complication rates have yet to be determined [44].

\section{Associated procedures and new perspectives in HTO}

Due to the better understanding of knee biomechanics and improved surgical techniques, indications for HTOs are increasing. Correct knee alignment has proven to be an essential factor in achieving good results with ligament, cartilage, and meniscal procedures.

Osteotomies can be indicated in the treatment of chronic ligamentous instability (alone or combined with ligament reconstruction/revision) or associated with chondral resurfacing and meniscal repair or transplant $[1 \bullet, 45,46]$.

Indications for HTO in patients with knee instability include $[1 \bullet]$ :

(1) Symptomatic anterior cruciate ligament (ACL) deficiency with symptomatic medial compartment arthritis/ overload.

(2) Chronic ACL deficiency with varus alignment and varus thrust.

(3) Chronic posterolateral instability/laxity with symptomatic varus hyperextension thrust.

(4) ACL reconstruction failure due to sagittal or coronal malalignment.

(5) Chronic posterior cruciate ligament (PCL) deficiency and symptomatic varus malalignment.

(6) In case of chronic medial collateral ligament (MCL) deficiency and valgus alignment, a varus HTO can be hypothesized, but a distal femoral osteotomy is generally preferred.

The goals of simultaneous HTO and ligament reconstruction are to correct malalignment, decrease knee joint load, and restore stability [46].
Physiological tibial slope ranges from $0^{\circ}$ to $18^{\circ}$ [47] and the slope can be modified with HTO [48]. The effect of tibial slope changes on anteroposterior knee stability has been described. When the tibial slope is decreased (as in closing wedge HTO or in opening wedge HTO with a posterior position of the spacer plate) the PCL is tensioned and the ACL is detensioned $[49,50]$. An opposite effect is obtained on the cruciate ligaments when the tibial slope is increased.

Many clinical trials have shown good results with combined ACL reconstruction and HTO [51-55].

Combined HTOs and biological surgical treatments (ie, articular cartilage surgery, meniscal repair, or transplantation) also showed good short- and mid-term results.

In the varus knee with medial compartment chondral lesions, an adequate correction of the limb alignment enhance tissue repair $[3 \cdot, 23]$. In addition, combined HTO and meniscal transplantation has become an accepted technique in young patients $(<40$ years $)$ with varus alignment and meniscal deficiency $[56,57]$.

Harris et al systematically reviewed 69 studies (4557 subjects) to evaluate the survival difference between isolated HTO and HTO with combined procedures. The mean follow-up was 7.1 years and the mean patient age was 53 years. At 5 years of follow-up, HTOs with articular cartilage surgery had significantly greater survival $(97.7 \%)$ than isolated HTO (92.4 \%), or HTO with meniscal allograft transplant $(90.9 \%)$. However, at all other time points, there was no significant survival difference between the groups [2].

Wong et al conducted a prospective randomized controlled clinical trial about of the intra-articular injection of cultured autologous bone marrow-derived mesenchymal stem cell combined with microfracture and medial opening wedge HTO. The authors concluded that at 2 years of follow-up the technique is effective in improving short-term clinical and radiological outcomes [58].

Ferruzzi et al compared clinical outcome in middle aged patients treated with isolated HTOs or HTOs associated with cartilage repair procedures (autologous chondrocyte implantation or microfractures). At 11 years of follow-up they obtained clinical and radiological improvement in all patients. HTOs and HTOs+autologous chondrocyte implantation groups obtained higher scores compared with HTOs+ microfractures group [3•].

HTO has also shown not to be contraindicated in high level athletes, alone, or combined with other procedures [43, 59].

\section{Knee arthroplasty and HTO}

Although both HTO and unicompartmental knee arthroplasty (UKA) are effective treatments for medial knee arthrosis and may delay or avoid the need for TKR, they should not be considered equivalent treatment options. When compared, 
both HTO and UKA showed satisfactory results and survival rates at mid- and long-term follow-up. Some authors described slightly better results for UKA [60-63], while others reported similar results for the 2 techniques [63-66].

However, when reviewing the literature, it becomes evident that HTO and UKA have different indications [67]. Only a small population of patients can be amenable to either HTO or UKA and this includes patients: (1) from 60 to 65 years old; (2) who are moderately active; (3) who are nonobese; (4) with mild varus malalignment (from $5^{\circ}$ to $10^{\circ}$ ); (5) without joint instability; (6) with a good range of motion; and (7) with moderate unicompartmental arthritis [67].

Given that TKR represents the endpoint of both HTO and UKA, similar survival time to TKR can be observed for the 2 techniques ( 9.7 years after HTO and 9.2 years after UKA) [62].

It has to be considered that revising UKA to TKR is more challenging than revising HTO to TKR. In the first case, bone loss needs to be managed during surgery [68], while HTO revision to TKR can be considered a primary TKR.

Although little data are available comparing opening or closing wedge HTO in terms of revision to TKR, opening wedge HTO has some theoretical advantages, including: no risk of patella alta, maintenance of bone stock, and lower risk of impingement between the tibial stem and the anterior tibial cortex.

Robertsson and Dahl compared the results and complications of primary TKR, HTO revision to TKR, and UKA revision to TKR. They found a risk of revision significantly higher after previous closing wedge HTO and UKA than de novo TKR, whereas open wedge HTO did not affect the outcome. Stemmed implants or special revision implants were used in $0.6 \%$ of the primary TKR, in $4 \%$ of the HTO to TKR conversions, and in $17 \%$ of the UKA to TKR conversions [69•].

\section{Conclusions}

Both lateral closing wedge and medial opening wedge HTOs have shown excellent clinical results and survival at short- and mid-term follow-up (Table 1). Many studies reported high survival rates not only after 5 years $(75 \%$ to $94 \%$ ) but also after 10 years ( $51 \%$ to $95 \%)$ of follow-up. Despite these encouraging short- and mid-term results, a decreasing rate of success and beneficial effects becomes evident after 15 years (39\% to $90 \%$ ) from surgery [7].

The role of these procedures is to unload the medial compartment of the knee delaying the progression of osteoarthritis.

According to literature, the ideal candidate for HTO is a young active patient ( $<65$ years), with medial isolated osteoarthritis (less than grade III according to Ahlbäck classification) and good range of motion $[1 \bullet, 15 \bullet, 64]$.
A careful preoperative planning is essential. Generally, a $5^{\circ}-6^{\circ}$ degree valgus overcorrection is planned [ $\left.1 \bullet\right]$. In young patients and athletes, overcorrection is not recommended and the goal is to achieve a neutral alignment [59], avoiding lateral overload.

Although no significant differences have been reported in the literature between opening and closing wedge HTOs, the author's preferred technique is opening wedge, which is easier, allows for multiplanar correction, and avoids tibiofibular joint disruption, compared with closing wedge technique.

HTO is an excellent surgical option in selected patients and can give good long-term follow-up outcomes. However, pooling the results is a challenge for the heterogeneity of data, variability of the patients enrolled, and the different scoring systems. Additional well-designed studies and long-term follow-up data are required to identify the ideal candidates and surgical techniques in HTO.

\section{Compliance with Ethics Guidelines}

Conflict of Interest Annunziato Amendola has received grants from the National Institutes of Health. Annunziato Amendola has received royalties from Arthrex, Inc and Arthrosurface, Inc. and owns stock in Arthrosurface Inc. and MTP Solutions.

Davide Bonasia, Giorgio Governale, Simone Spolaore, and Roberto Rossi declare that they have no conflict of interest.

Human and Animal Rights and Informed Consent This article does not contain any studies with human or animal subjects performed by any of the authors.

\section{References}

Papers of particular interest, published recently, have been highlighted as:

- Of importance

1. Rossi R, Bonasia DE, Amendola A. The role of high tibial osteotomy in the varus knee. J Am Acad Orthop Surg. 2011;19: 590-9. Note the preoperative planning calculations.

2. Harris JD, McNeilan R, Siston RA, Flanigan DC. Survival and clinical outcome of isolated high tibial osteotomy and combined biological knee reconstruction. Knee. 2013;20:154-61. doi:10. 1016/j.knee.2012.12.012 [Epub Mar 9, 2013].

3. Ferruzzi A, Buda R, Cavallo M, Timoncini A, Natali S, Giannini S. Cartilage repair procedures associated with high tibial osteotomy in varus knees: clinical results at 11 years' follow-up. Knee. 2014;21: 445-50. [Epub Nov 27, 2013]. doi:10.1016/j.knee.2013.11.013. Combination of HTO and cartilage resurfacing is described.

4. Aglietti P, Rinonapoli E, Stringa G, Taviani A. Tibial osteotomy for the varus osteoarthritic knee. Clin Orthop Relat Res. 1983;176:239-51.

5. Naudie D, Bourne RB, Rorabeck $\mathrm{CH}$, Bourne TJ. The Install Award. Survivorship of the high tibial valgus osteotomy. A 10 to 22-year follow-up study. Clin Orthop Relat Res. 1999;367:18-27. 
6. Rudan JF, Simurda MA. High tibial osteotomy. A prospective clinical and roentgenographic review. Clin Orthop Relat Res. 1990;255:251-6.

7. Amendola A, Bonasia DE. Results of high tibial osteotomy: review of the literature. Int Orthop. 2010;34:155-60.

8. Schallberger A, Jacobi M, Wahl P, Maestretti G, Jakob RP. High tibial valgus osteotomy in unicompartmental medial osteoarthritis of the knee: a retrospective follow-up study over 13-21 years. Knee Surg Sports Traumatol Arthrosc. 2011;19:122-7. doi:10.1007/ s00167-010-1256-4 [Epub Sep 7, 2010].

9. Niemeyer P, Schmal H, Hauschild O, von Heyden J, Südkamp NP, Köstler W. Open-wedge osteotomy using an internal plate fixator in patients with medial-compartment gonarthritis and varus malalignment: 3-year results with regard to preoperative arthroscopic and radiographic findings. Arthroscopy. 2010;26:1607-16. doi:10.1016/j.arthro.2010.05. 006. This paper helps understanding arthroscopic prognostic factors before HTO.

10. Bode G, von Heyden J, Pestka J, Schmal H, Salzmann G, Südkamp $\mathrm{N}$, et al. Prospective 5-year survival rate data following open-wedge valgus high tibial osteotomy. Knee Surg Sports Traumatol Arthrosc. 2013. [Epub ahead of print].

11. Schröter S, Gonser CE, Konstantinidis L, Helwig P, Albrecht D. High complication rate after biplanar open wedge high tibial osteotomy stabilized with a new spacer plate (Position HTO plate) without bone substitute. Arthroscopy. 2011;27:644-52. doi:10. 1016/j.arthro.2011.01.008.

12. Hernigou $\mathrm{P}$, Roussignol $\mathrm{X}$, Flouzat-Lachaniette $\mathrm{CH}$, Filippini $\mathrm{P}$, Guissou I, Poignard A. Opening wedge tibial osteotomy for large varus deformity with Ceraver resorbable beta tricalcium phosphate wedges. Int Orthop. 2010;34:191-9. doi:10.1007/s00264-0090875-1 [Epub Oct 1, 2009].

13. DeMeo PJ, Johnson EM, Chiang PP, Flamm AM, Miller MC. Midterm follow-up of opening-wedge high tibial osteotomy. Am J Sports Med. 2010;38:2077-84. doi:10.1177/0363546510371371 [Epub Jul 1, 2010].

14. Laprade RF, Spiridonov SI, Nystrom LM, Jansson KS. Prospective outcomes of young and middle-aged adults with medial compartment osteoarthritis treated with a proximal tibial opening wedge osteotomy. Arthroscopy. 2012;28:354-64. doi:10.1016/j.arthro. 2011.08.310 [Epub Dec 14, 2011].

15. Bonasia DE, Dettoni F, Sito G, Blonna D, Marmotti A, Bruzzone $\mathrm{M}$, et al. Medial opening wedge high tibial osteotomy for medial compartment overload/arthritis in the varus knee: prognostic factors. Am J Sports Med. 2014;42: 690-8. [Epub Jan 21, 2014]. doi:10.1177/0363546513516577. This paper describes pre- and postoperative prognostic factors of opening wedge HTO.

16. Niinimäki TT, Eskelinen A, Mann BS, Junnila M, Ohtonen P, Leppilahti J. Survivorship of high tibial osteotomy in the treatment of osteoarthritis of the knee: finnish registry-based study of 3195 knees. J Bone Joint Surg (Br). 2012;94:1517-21. doi:10.1302/ 0301-620X.94B11.29601. This paper describes the results of HTO from a national registry.

17. Flecher X, Parratte S, Aubaniac JM, Argenson JN. A 12-28-year follow-up study of closing wedge high tibial osteotomy. Clin Orthop Relat Res. 2006;452:91-6.

18. Akizuki S, Shibakawa A, Takizawa T, Yamazaki I, Horiuchi H. The long-term outcome of high tibial osteotomy: a ten- to 20-year follow-up. J Bone Joint Surg (Br). 2008;90:592-6. doi:10.1302/ 0301-620X.90B5.20386.

19. Gstöttner M, Pedross F, Liebensteiner M, Bach C. Long-term outcome after high tibial osteotomy. Arch Orthop Trauma Surg. 2008;128:111-5.

20. Hui C, Salmon LJ, Kok A, Williams HA, Hockers N, van der Tempel WM, et al. Long-term survival of high tibial osteotomy for medial compartment osteoarthritis of the knee. Am J Sports Med. 2011;39:64 70. Epub Sep 10, 2010]. doi:10.1177/0363546510377445.

21. Efe T, Ahmed G, Heyse TJ, Boudriot U, Timmesfeld N, FuchsWinkelmann S, et al. Closing-wedge high tibial osteotomy: survival and risk factor analysis at long-term follow-up. BMC Musculoskelet Disord. 2011;12:46. doi:10.1186/1471-2474-12-46.

22. Jackson JP, Waugh W. Tibial osteotomy for osteoarthritis of the knee. J Bone Joint Surg (Br). 1961;43-B:746-51.

23. Coventry MB. Osteotomy of the upper portion of the tibia for degenerative arthritis of the knee: a preliminary report. J Bone Joint Surg Am. 1965;47-A:984-90.

24. Atrey A, Morison Z, Tosounidis T, Tunggal J, Waddell JP. Complications of closing wedge high tibial osteotomies for unicompartmental osteoarthritis of the knee. Bone Joint Res. 2011;1:205-9.

25. Huang Y, Gu J, Zhou Y, Li Y. Osteotomy at the distal third of tibial tuberosity with LCP L-buttress plate for correction of tibia vara. J Orthopaed Surg Res. 2014;9:9.

26. Spahn G. Complications in high tibial (medial opening wedge) osteotomy. Arch Orthop Trauma Surg. 2004;124: 649-53.

27. Smith TO, Sexton D, Mitchell P, Hing CB. Opening- or closingwedged high tibial osteotomy: a meta-analysis of clinical and radiological outcomes. Knee. 2011;18:361-8. doi:10.1016/j.knee. 2010.10.001 [Epub Oct 29, 2010].

28. Brouwer RW, Bierma-Zeinstra SMA, Raaij TM, et al. Osteotomy for medial compartment arthritis of the knee using a closing wedge or an opening wedge controlled by a Puddu Plate. J Bone Joint Surg (Br). 2006;88-B:1454-9.

29. Lobenhoffer P, Agneskirchner JD. Improvements in surgical technique of valgus high tibial osteotomy. Knee Surg Sports Traumatol Arthrosc. 2003;11:132-8.

30. Seagrave RA, Sojka J, Goodyear A, Munns SW. Utilizing reamer irrigator aspirator (RIA) autograft for opening wedge high tibial osteotomy: a new surgical technique and report of three cases. Int J Surg Case Rep. 2014;5:37-42. doi:10.1016/j.ijscr.2013.11.004 [Epub Nov 15, 2013].

31. Kuremsky MA, Schaller TM, Hall CC, Roehr BA, Masonis JL. Comparison of autograft vs allograft in opening-wedge high tibial osteotomy. J Arthroplasty. 2010;25:951-7. doi:10.1016/j.arth.2009. 07.026 [Epub Sep 23, 2009].

32. Aryee $\mathrm{S}$, Imhoff $\mathrm{AB}$, Rose $\mathrm{T}$, Tischer $\mathrm{T}$. Do we need synthetic osteotomy augmentation materials for opening wedge high tibial osteotomy. Biomaterials. 2008;29:3497-502.

33. Hernigou $\mathrm{P}, \mathrm{Ma} \mathrm{W}$. Open wedge tibial osteotomy with acrylic bone cement as bone substitute. Knee. 2001;8:103-10.

34. Dallari D, Savarino L, Stagni C, Cenni E, Cenacchi A, Fornasari PM, et al. Enhanced tibial osteotomy healing with use of bone grafts supplemented with platelet gel or platelet gel and bone marrow stromal cells. J Bone Joint Surg Am. 2007;89:2413-20.

35. Kawaguchi H, Jingushi S, Izumi T, Fukunaga M, Matsushita T, Nakamura T, et al. Local application of recombinant human fibroblast growth factor- 2 on bone repair: a dose-escalation prospective trial on patients with osteotomy. J Orthop Res. 2007;25:480-7.

36. Peerbooms JC, Colaris JW, Hakkert AA, Van Appeldorn M, Bruijn DJ, Den Oudsten BL, et al. No positive bone healing after using platelet rich plasma in a skeletal defect. An observational prospective cohort study. Int Orthop. 2012;36:2113-9. doi:10.1007/ s00264-012-1603-9 [Epub Jun 26, 2012].

37. Griffin XL, Wallace D, Parsons N, Costa ML. Platelet rich therapies for long bone healing in adult. Cochrane Database System Rev. 2012;7: CD009496.

38. Pape D, Lorbach O, Schmitz C, Busch LC, Van Giffen N, Seil R, et al. Effect of a biplanar osteotomy on primary stability following high tibial osteotomy: a biomechanical cadaver study. Knee Surg Sports Traumatol Arthrosc. 2010;18:204-11. 
39. Agneskirchner JD, Freiling D, Hurschler C, Lobenhoffer P. Primary stability of four different implants for opening wedge high tibial osteotomy. Knee Surg Sports Traumatol Arthrosc. 2006;14:291300 .

40. Dorsey WO, Miller BS, Tadje JP, Bryant CR. The stability of three commercially available implants used in medial opening wedge high tibial osteotomy. J Knee Surg. 2006;19:95-8.

41. Spahn G, Mückley T, Kahl E, Hofmann GO. Biomechanical investigation of different internal fixations in medial opening wedge high tibial osteotomy. Clin Biomech (Bristol, Avon). 2006;21:272-8

42. Pape D, Kohn D, van Giffen N, Hoffmann A, Seil R, Lorbach O. Differences in fixation stability between spacer plate and plate fixator following high tibial osteotomy. Knee Surg Sports Traumatol Arthrosc. 2013;21:82-9. doi:10.1007/s00167-0111693-8 [Epub Oct 9, 2011].

43. Scordino LE, DeBerardino TM. Surgical treatment of osteoarthritis in the middle-aged athlete: new horizons in high tibial osteotomies. Sports Med Arthrosc. 2013;21:47-51. doi:10.1097/JSA. 0b013e318278f71d.

44. Cotic M, Vogt S, Hinterwimmer S, Feucht MJ, Slotta-Huspenina J, Schuster T, et al. A matched-pair comparison of two different locking plates for valgus-producing medial open-wedge high tibial osteotomy: peek-carbon composite plate vs titanium plate. Knee Surg Sports Traumatol Arthrosc. 2014. [Epub ahead of print].

45. McNamara I, Birmingham TB, Fowler PJ, Griffin JR. High tibial osteotomy: evolution of research and clinical applications-a Canadian experience. Knee Surg Sports Traumatol Arthrosc. 2013;21:23-31.

46. Naudie DD, Amendola A, Fowler PJ. Opening wedge high tibial osteotomy for symptomatic hyperextension-varus thrust. Am J Sports Med. 2004;32:60-70.

47. Genin P, Weill G, Julliard R. The tibial slope: proposal for a measurement method [French]. J Radiol. 1993;74:27-33.

48. Ducat A, Sariali E, Lebel B, Mertl P, Hernigou P, Flecher X, et al. Posterior tibial slope changes after opening- and closing-wedge high tibial osteotomy: a comparative prospective multicenter study. Orthop Traumatol Surg Res. 2012;98:68-74.

49. Lerat JL, Moyen B, Garin C, Mandrino A, Besse JL, Brunet-Guedj E. Anterior laxity and internal arthritis of the knee. Results of the reconstruction of the anterior cruciate ligament associated with tibial osteotomy. Rev Chir Orthop Reparatrice Appar Mot. 1993;79:365-74.

50. Hohmann E, Bryant A, Imhoff AB. The effect of closed wedge high tibial osteotomy on tibial slope: a radiographic study. Knee Surg Sports Traumatol Arthrosc. 2006;14:454-9.

51. Dejour H, Neyret P, Boileau P, Donell ST. Anterior cruciate reconstruction combined with valgus tibial osteotomy. Clin Orthop Relat Res. 1994;299:220-8.

52. Williams III RJ, Kelly BT, Wickiewicz TL, Altchek DW, Warren RF. The short-term outcome of surgical treatment for painful varus arthritis in association with chronic ACL deficiency. J Knee Surg. 2003;16:9-16.

53. Bonin N, Ait Si Selmi T, Donell ST, Dejour H, Neyret P. Anterior cruciate reconstruction combined with valgus upper tibial osteotomy: 12 years follow-up. Knee. 2004;11:431-7.
54. Boss A, Stutz G, Oursin C, Gächter A. Anterior cruciate ligament reconstruction combined with valgus tibial osteotomy (combined procedure). Knee Surg Sports Traumatol Arthrosc. 1995;3:187-91.

55. Trojani C, Elhor H, Carles M, Boileau P. Anterior cruciate ligament reconstruction combined with valgus high tibial osteotomy allows return to sports. Ortop Traumatol Surg Res. 2014;100:209-12.

56. Bonasia DE, Amendola A. Combined medial meniscal transplantation and high tibial osteotomy. Knee Surg Sports Traumatol Arthrosc. 2010;18:870-3.

57. Amendola A. Knee osteotomy and meniscal transplantation: indications, technical considerations, and results. Sports Med Arthrosc. 2007;15:32-8.

58. Wong KL, Lee KB, Tai BC, Law P, Lee EH, Hui JH. Injectable cultured bone marrow-derived mesenchymal stem cells in varus knee with cartilage defects undergoing high tibial osteotomy: a prospective, randomized controlled clinical trial with 2 years' follow-up. Arthroscopy. 2013;29:2020-8.

59. Warme BA, Aalderink K, Amendola A. Is there a role for high tibial osteotomies in the athlete? Sports Health. 2011;3:59-69.

60. Broughton NS, Newman JH, Baily RA. Unicompartmental replacement and high tibial osteotomy for osteoarthritis of the knee. A comparative study after 5-10 years' follow-up. J Bone Joint Surg (Br). 1986;68:447-52.

61. Stukenborg-Colsman C, Wirth CJ, Lazovic D, Wefer A. High tibial osteotomy vs unicompartmental joint replacement in unicompartmental knee joint osteoarthritis: 7-10-year follow-up prospective randomised study. Knee. 2001;8:187-94.

62. Spahn G, Hofmann GO, von Engelhardt LV, Li M, Neubauer H, Klinger HM. The impact of high tibial valgus osteotomy and unicondylar medial arthroplasty on the treatment for knee osteoarthritis: a meta-analysis. Knee Surg Sports Traumatol Arthrosc. 2013;21:96-112.

63. Brouwer RW, Raaij TM, Bierma-Zeinstra SM, Verhagen AP, Jakma TS, Verhaar JA. Osteotomy for treating knee osteoarthritis. Cochrane Database Syst Rev. 2007;18:CD004019.

64. Dettoni F, Maistrelli GL, Rossi P, Castoldi F, Stojimirovich D, Rossi R. UKA vs HTO: clinical results at short term follow-up. 75th AAOS Annual Meeting. San Francisco, CA; 2008.

65. Yim JH, Song EK, Seo HY, Kim MS, Seon JK. Comparison of high tibial osteotomy and unicompartmental knee arthroplasty at a minimum follow-up of 3 years. J Arthroplasty. 2013;28:243-7.

66. Fu D, Li G, Chen K, Zhao Y, Hua Y, Cai Z. Comparison of high tibial osteotomy and unicompartmental knee arthroplasty in the treatment of unicompartmental osteoarthritis: a meta-analysis. J Arthroplasty. 2013;28:759-65.

67. Dettoni F, Bonasia DE, Castoldi F, Bruzzone M, Blonna D, Rossi R. High tibial osteotomy vs unicompartmental knee arthroplasty for medial compartment arthrosis of the knee: a review of the literature. Iowa Orthop J. 2010;30:131-40.

68. Gill T, Schemitsch EH, Brick GW, Thornhill TS. Revision total knee arthroplasty after failed unicompartmental knee arthroplasty or high tibial osteotomy. Clin Orthop Relat Res. 1995;321:10-8.

69. Robertsson O, W-Dahl A. The risk of revision after TKA is affected by previous HTO or UKA. Clin Orthop Relat Res. 2014. [Epub ahead of print] Important paper describing the risks of revision HTO and UKA to TKR. 